\title{
Validity and Reliability of the Turkish Version of the Family Empowerment Scale for Caregivers of Adults with Mental Health Issues (FES-AMT)
}

\author{
Hatice Durmaz ${ }^{1(\underline{I D})}$, Esra Yildiz ${ }^{2(\underline{I D})}$
}

\author{
${ }^{1}$ Department of Pysciatric Nursing, Ataturk University Faculty of Nursing, Erzurum, Turkey \\ ${ }^{2}$ Department of Public Health Nursing, Ataturk University Faculty of Nursing, Erzurum, Turkey
}

Copyright@ Author(s) - Available online at https://dergipark.org.tr/en/pub/mbsjohs

Content of this journal is licensed under a Creative Commons Attribution-NonCommercial 4.0 International

License,

Received: 29 June 2021, Accepted: 10 November 2021, Published online: 31 December 2021

(C) Ordu University Institute of Health Sciences, Turkey, 2021

\begin{abstract}
Objective: This study aimed to adapt the Family Empowerment Scale for family caregivers of adults with mental health issues to the Turkish context.

Methods: The study data is collected from 1 January 2019 to 15 April 2020. The FES was originally developed by Koren et al.and, Kageyama et al. adapted it to assess the empowerment of family caregivers of adults with mental health issues, and they tested its validity and reliability. The study sample comprised 223 caregivers whose families of patients staying at the psychiatry clinic of a research and training hospital and those who had applied to the polyclinic. The scale has 34 items rated on a 5-point Likert-type scale. The scoring was subjected to a two-phase cluster analysis to obtain detailed information about the caregiver's empowerment.

Results: Confirmatory factor analysis was applied to examine the goodness of fit and construct validity of the structure, which was determined to consist of 3 factors by explanatory factor analysis. Factor loading range from 0.722 (item 20) to 0.008 (item 22). Exploratory factor analysis was conducted. Kaiser - Meyer Olkin sampling adequacy value was determined as 0.894. The Cronbach's alpha coefficient of internal consistency was .908. The scale has three subscales: Family, Service System, and Community/Political. The total variance explained by the scale was $47.78 \%$. The Cronbach's alpha coefficient for the whole scale was .927.

Conclusion: The Turkish version of the Family Empowerment Scale for caregivers of adults with mental health issues (FES-AMT) is a valid and reliable measurement tool. This scale can be used to evaluate patient relatives in the clinical practice of nurses.
\end{abstract}

Key words: Family, Empowerment, Turkish, Validity

Suggested Citation: Durmaz H, Yildiz E. Validity and Reliability of the Turkish Version of the Family Empowerment Scale for Caregivers of Adults with Mental Health Issues (FES-AMT). Mid Blac Sea Journal of Health Sci, 2021; 7(3):348-357.

Address for correspondence/reprints:

Hatice Durmaz

Telephone number: +90 (530) 9218527

E-mail: haticedurmaz_25@hotmail.com

\section{Introduction}

Research emphasizing the difficulties experienced by primary caregivers of individuals with chronic diseases has increased (1). Family empowerment is gaining importance, especially in situations that cause disability (2). Empowerment is an important concept for the status of parents within health care services (1) because family empowerment has been shown to 
have a positive effect on different patient populations in various cultures $(1,3-5)$ Researchers have used various approaches and measurement tools to assess family empowerment (6). In cases of psychiatric disorders, family caregiver empowerment is a significant factor in coping with the nature of the disease. Family empowerment in social, political, and service-related matters can facilitate the work of caregivers $(7,8)$.

Additionally, the empowerment of family caregivers has emerged as a new objective of family interventions. This includes disease management required for patient care as well as finding social roles through interaction with other family caregivers and participating in advocacy (7). The 34-item Family Empowerment Scale (FES) was developed by Koren, DeChillo, and Friesen in 1992 to measure empowerment among the parents of children with emotional disability. The reliability and validity of this scale have been verified in various languages and cultures (7-10). The FES has been used and validated in various populations, including children with diabetes (1,9). Kageyama et al. (7) developed a Japanese version of the FES for family caregivers of adult patients with mental disorders and verified its validity and reliability (7). Due to the nature of psychiatric patients' illnesses, strong caregivers are essential for care. In some cases, the caregiver needs to make a decision instead of the patient. In order to use this authority, the caregiver must be strong. Therefore, there is a need for a measurement tool to evaluate the strengths of caregivers, especially of psychiatric patients.

No studies have assessed the empowerment of family caregivers in the Turkish context. Thus, this study aimed to adapt the FES for caregivers of adults with mental health issues to the Turkish context and verify its validity and reliability.

\section{Methods}

This methodological study was conducted to adapt the FES for caregivers of adults with mental health issues to the Turkish context and test its validity and reliability. The study was conducted in three stages: 1) translation of the scale into Turkish and backtranslation into English, 2) assessment of the content validity of the scale by a group of experts, and 3) psychometric analyses (factor analysis and calculation of Cronbach's alpha, validity coefficient, and fit indices).

Erzurum is located east of Turkey. The hospital where the research was conducted provides services to patients in both the rural areas and the city center of 11 cities located around Erzurum. It is close to the
Iranian border. The city has an average population of 762,000 . The study sample comprised the families of patients staying at the psychiatry clinic of a research and training hospital and those who had applied to the polyclinic. To adapt a scale to another culture, the sample should be at least 5 to 10 times larger than the number of scale items. Therefore, this study was carried out with 223 caregivers. The study data were collected from 1 January 2019 to 15 April 2020, after obtaining approval from the ethics committee of the local and official ethics committee.

The study included caregivers of patients diagnosed with a psychiatric disorder who had no problem communicating. Data were collected using the Turkish version of the Family Empowerment Scale for Caregivers of Adults with Mental Health Issues through face-to-face interviews.

\section{Data collection tools}

Data were collected through face to face using the Turkish version of the FES for caregivers of adults with mental health issues. The authors obtained permission from Kageyama, Koren, DeChillo, and Friesen to develop a Turkish version of their scale.

\section{Evaluation of data}

Data were analyzed using SPSS and AMOS software. The study used exploratory factor analysis to test the structural validity and confirmatory factor analysis to test the accuracy of the factor structure. Fit indices and Cronbach's alpha coefficients were calculated.

\section{FES for caregivers of adults with mental health issues}

The FES was originally developed by Koren et al. (2) to assess the empowerment of parents of children with mental health issues (2). Kageyama et al. (6) adapted it to the Japanese context to assess the empowerment of family caregivers of adults with mental health issues, and they tested its validity and reliability (7). They changed "child" to "the person," "children" to "people with disorders," and "parent" to "family" (referring to the family caregiver). In addition, "grow and develop" was changed to "recovery" (items 4 and 27), and "special education laws" was revised to "the law related to the disorders" (item 24). However, the dimensions of the Japanese version are the same as those of the original scale. The FES divides empowerment into two dimensions. The first refers to the levels of empowerment: service system and community/political. The second refers to how empowerment is expressed in the form of attitudes and knowledge. 
Each item is scored on a Likert-type scale from 1 (not true at all) to 5 (very true). The FES assesses empowerment based on three subscales: Family (12 items), Service System (12 items), and Community/Political (10 items). See Table 1

Table 1. Dimensions of the Family Empowerment Scale

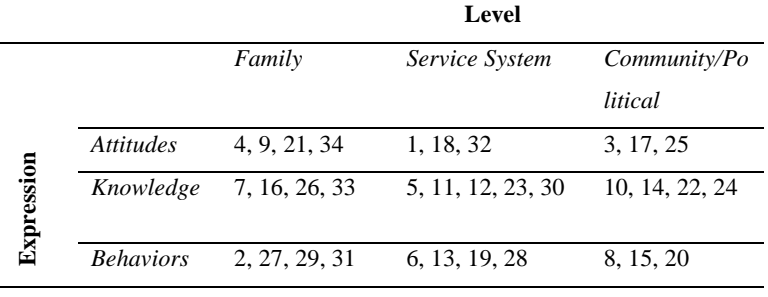

The Family subscale assesses parents' management of everyday matters in the home. Service System refers to the professionals and institutions providing services to the patient; this subscale primarily concerns parents collaborating with the service system to provide sufficient care for their children. The Community/Political subscale refers to legislative bodies, policymakers, agencies, and community members; it primarily concerns caregivers' advocacy for the relevant population.

The mean score of each subscale is calculated by summing the scores for each item and dividing the total score by the number of questions. Although all subscale scores can be added to obtain a total score ranging from 3 to 15 , it is recommended that each subscale score be used since they measure different areas.

The language validity of the scale was examined, and the scale was then translated into Turkish and reviewed by eight health professionals who speak English as a second language. The content validity index was calculated as 0.80 .

\section{Statistical analysis}

Data were analyzed using SPSS and AMOS software. The study used exploratory factor analysis to test the structural validity and confirmatory factor analysis to test the accuracy of the factor structure. Fit indices and Cronbach's alpha coefficients were calculated.

\section{Results}

Participants' sociodemographic characteristics

The study was conducted with 223 participants. Their mean age was $39.70 \pm 11.42$. Ninety participants $(52.5 \%)$ were male, $76(34.1 \%)$ were bachelor graduates, 170 (76.2) were married and 125 (56.1\%) had a job. Participants are spouses, siblings or parents of people with mental illness.
Factor analysis

Exploratory factor analysis was conducted. Kaiser - Meyer - Olkin sampling adequacy value was determined as 0.894. As Bartlett's significance test level is $\mathrm{p}<0.05$ and the KMO coefficient approaches are 1, the sample should be considered sufficient. The scale was weighted in three subscales and explained $47.78 \%$ of the total variance.

We did rotated component matrix (Table 2). Varimax rotation was used to determine the factors that scale items weighted.

Table 2. Factor loading of the Turkish version of the Family Empowerment Scale for caregivers of adults with mental health issues

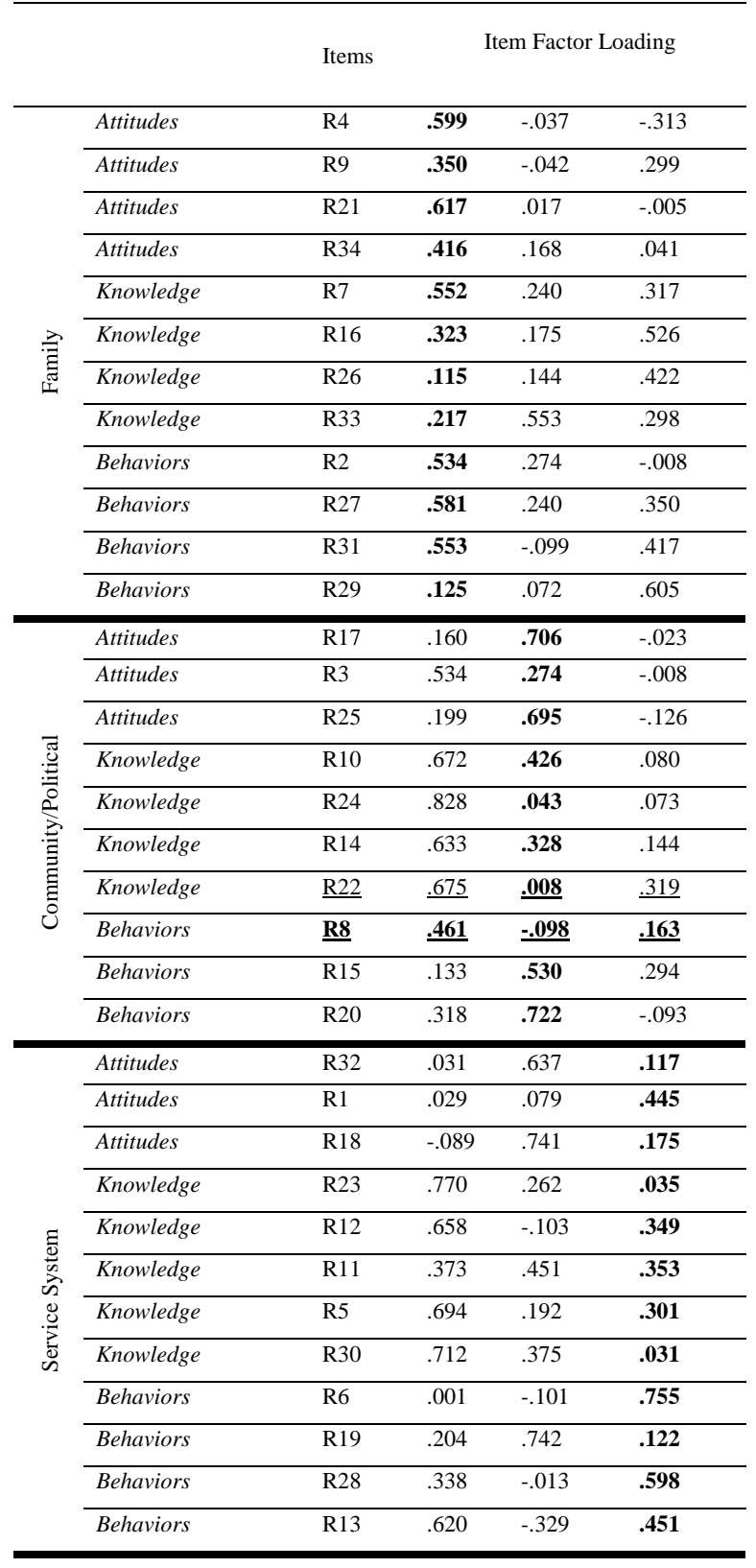


Factor loads are between -0.098 and 0.722 . All items other than items 8 was positively weighted in the same subscales as in the original scale. Item 8 of the Community/Political subscale were negatively weighted. These three items were positively weighted in the family subscale. Factor loading range from 0.722 (item 20) to 0.008 (item 22) (Table 3).

Table 3. Fit indices of the Turkish version of the Family Empowerment Scale for caregivers of adults with mental health issues

\begin{tabular}{cc}
\hline Acceptable Fit Indices & Measured Fit Indices \\
\hline$\chi 2 /$ sd $<5$ & 3,745 \\
GFI $>0.90$ & 0.936 \\
AGFI $>0.90$ & 0.910 \\
CFI $>0.90$ & 0.912 \\
TLI $>0.90$ & 0.931 \\
RMSEA $<0.08$ & 0.077 \\
\hline
\end{tabular}

Confirmatory Factor Analysis is an extension of explanatory factor analysis that evaluates the underlying structure of the data. Exploratory Factor Analysis tries to provide a determination function, to obtain information for establishing a hypothesis. Confirmatory Factor Analysis is used to test whether there is a sufficient relationship between these determined factors. In addition, Confirmatory Factor Analysis is used to test which variables are related to which factors, whether the factors are independent from each other, and whether the factors are sufficient to explain the model. Confirmatory factor analysis was applied to examine the goodness of fit and construct validity of the structure, which was determined to consist of 3 factors by explanatory factor analysis. Fit index analyses showed that the model was compatible with the data, as $\chi 2 / \mathrm{df}$ was lower than 5, indicating a good model fit. The goodness-of-fit index and Tucker-Lewis index were above 0.90 , the adjusted goodness-of-fit index was above 0.85 , and the root mean square error of approximation was below 0.08 , indicating an acceptable model fit.

\section{Reliability}

The Cronbach's alpha coefficients were 0.736 for the Family subscale, 0.905 for the Service System subscale, and 0.773 for the Community/Political subscale. The Cronbach's alpha coefficient for the whole scale was 0.927 Cronbach alpha values above 0.70 value. These values indicate a good level of reliability.

\section{Discussion}

This study aimed to determine the validity and reliability of the Turkish version of the FES for family caregivers of adults with mental health issues (FESAMT).

Factor analysis was conducted to search for correlations between the variables. The result of Bartlett's test was significant, indicating that the variables were correlated. The data had multiple normal distributions. The KMO value was above 0.80 , which is excellent for a sample of this size (11).

In this study, we found that many items were not weighted at the required subscale. Items 8, 22 and 24 were the lowest-weight items. However, we found that items other than item 8 were not weighted negatively. For this reason, we did not remove items from the scale. The scale items 8,22 , and 24 were weighted differently compared to the original scale. Kageyama et al. (7) found that the weighting of these items differed on community-political subscale in the original scale (7). Koren et al. (2) found that items 22 and 24 were weighted higher than .40 on service system and community-political subscales of the original scale (2). Items 8 and 22 featured the word "legislator." Koren et al. (2) stated that these items are relevant to parents who can contact legislators and make a legal complaint. However, Kageyama et al. (7) cautioned that the Japanese may have difficulty understanding these two items because they are unlikely to encounter legislators (7). The results of the present study show similarities between the Turkish and Japanese contexts. Like the Japanese, Turkish people rarely have the opportunity to meet legislators. Items 8, 22, and 24 were modified in studies conducted with the parents of child patients $(1,4)$. These items should also be modified for validity and reliability studies among family caregivers of adult patients. Item 24 involves knowing the rights of people and families who are subject to laws on disorders. This result showed that Turkish people may be not aware of their rights. And item 26 has lower factor loading. It involves being able to ask for help from others when assistance with family problems is needed. Turkish people cannot ask for help from others when it comes to mental illnesses. These findings showed that up to us who care nurses in psychiatric nursing in Turkey demonstrates the need for more strength in the policy areas of the patients' relatives. Nurses can increase the effectiveness of their interventions by knowing the areas where their relatives need to be strengthened. These results, obtained in Turkey reveals similarities to Japanese and American culture. These similarities in societies also present the commonness of 
international problems to nurses in evaluating patient relatives. These problems can also provide a source of data for intercultural nursing.

Also, Segers et. all. (1) In the validity and reliability study of the scale in the Netherlands, they found that there were differences in understanding in the translation of the items in our scale into a new language. For example, they described the term service system in item 23 as incomprehensible (1). This finding; suggested that this may be the reason for the low factor loadings of some items in our study. According to Zolmajd et al. (8) found that the factor loads of similar items of the scale were low. Similar to the results of this study, the factor load of the items is below .30. This finding may suggest that the Persian culture and the region where this study was conducted show similarities (8).

In fact, since the sub-dimensions of the items of the scale were predetermined, we focused on the fit index data for validity. We can say that the scale has validity because the fit index data are acceptable. According to the fit indices from the confirmatory factor analysis, the scale has a good level of validity. Therefore, it can be concluded that the Turkish version of the FES is a valid measurement tool.

The fact that the $\chi^{2}$ / sd value is below 5 indicates that the data obtained and the model fit are acceptable. The GFI value that obtained in this study is a good fit value. This value is a measure of the amount of variance and covariance that can be explained by the model. AGFI is a criterion sensitive to the sample volume. The AGFI value that obtained indicates perfect fit. The CFI value shows the mismatch between the data and the hypothetical model. The CFI value that obtained from this study is a good fit value. The TLI value and RMSA values also show a good fit (12).

The Cronbach's alpha coefficients were calculated to test the reliability of the scale. The Cronbach's alpha coefficients for the whole scale and the three subscales were above .70 , similar to the results of Kageyama et al. (7). This indicates that the Turkish version of the scale is a reliable measurement tool (7). The high reliability of the scale indicates that this scale can be used by psychiatric nurses to evaluate caregivers

\section{Implications for caregiving}

- Empowerment of families that care for adults with mental health problems has increased interest in among mental health nurses in Turkey. Even this interest has even turned into a purpose for planned interventions for families.
- The scale is suitable for all families that have adults with mental health problems with inpatient or outpatient treatment.

- It is easy to apply, understandable and purposeful.

- The scale offers tips to families for reducing of caregiving burdenLimitations and future researches

\section{Conclusion}

The Turkish version of the FES for family caregivers of adults with mental health issues is a valid and reliable measurement tool. Further research could also verify the validity and reliability of the scale for the family caregivers of children.

Ethics Committee Approval: Approval from was received the ethics committee of the nursing department of Atatürk University (2018-5/2).

Peer-review: Externally peer-reviewed.

\section{Author Contributions:}

Concept: H.D, E.Y, Design: H.D, E.Y; Literature search: H.D, E.Y, Data Collection and Processing: H.D, Analysis or Interpretation: E.Y, Writing: H.D, E.Y

Conflict of Interest: No conflict of interest was declared by the authors.

Financial Disclosure: The authors declared that this study hasn't received no financial support.

\section{References}

1. Segers EW, van den Hoogen A, van Eerden IC, Hafsteinsdóttir T, Ketelaar M. Perspectives of parents and nurses on the content validity of the Family Empowerment Scale for parents of children with a chronic condition: A mixedmethods study. Child: care, health and development. 2019;45(1):111-20.

2. Koren PE, DeChillo N, Friesen BJ. Measuring empowerment in families whose children have emotional disabilities: a brief questionnaire. Rehabilitation psychology. 1992;37(4):305.

3. Chiocchi J, Lamph G, Slevin P, Fisher-Smith D, Sampson M. Can a carer (peer) led psychoeducation programme improve mental health carers well-being, reduce burden and enrich empowerment: a service evaluation study. The Journal of Mental Health Training, Education and Practice. 2019;14(2):131-40. 
4. Hayslip Jr B, Smith GC, Montoro-Rodriguez J, Streider FH, Merchant W. The utility of the family empowerment scale with custodial grandmothers. Journal of Applied Gerontology. 2017;36(3):32050.

5. Vuorenmaa M, Halme N, Åstedt-Kurki P, Kaunonen M, Perälä ML. The validity and reliability of the $\mathrm{F}$ innish $\mathrm{F}$ amily $\mathrm{E}$ mpowerment $\mathrm{S}$ cale (FES): a survey of parents with small children. Child: care, health and development. 2014;40(4):597-606.

6. Kim J, Bryan J. A first step to a conceptual framework of parent empowerment: Exploring relationships between parent empowerment and academic performance in a national sample. Journal of Counseling \& Development. 2017;95(2):168-79.

7. Kageyama M, Nakamura $\mathrm{Y}$, Kobayashi $\mathrm{S}$, Yokoyama K. Validity and reliability of the Family Empowerment Scale for caregivers of adults with mental health issues. Journal of psychiatric and mental health nursing. 2016;23(8):521-31.

8. Zolmajd Z. Psychometric properties of the Persian version of the family empowerment scale. Iranian Journal of Psychiatry and Clinical Psychology. 2018;24(2):216-29.

9. Florian V, Elad D. The Impact of Mothers' Sense of Empowerment oh the Metabolic Control of Their Children With Juvenile Diabetes. Journal of Pediatric Psychology. 1998;23(4):239-47.

10. Van Eerden I. Evaluating content validity of the Dutch translation of the Family Empowerment Scale in the Neonatal Intensive Care Unit using mixed methods 2017.

11. Yalcin K. SPSS and AMOS Applied Statistical Analysis. Ankara: Nobel Publisher; 2016.

12.Harrington D. Confirmatory factor analysis: Oxford university press; 2009. 